\title{
Supporting Information \\ Development of an Aerosol Chemiluminescent \\ Detector Coupled to Capillary Electrophoresis for \\ Saccharides Analysis
}

Guangming Huang, Yi Lv, Sichun Zhang, Chengdui Yang and Xinrong Zhang*

Department of Chemistry, Key Laboratory for Atomic and Molecular Nanosciences of

Education Ministry, Tsinghua University, 100084, Beijing, P. R. China

Corresponding Author: xrzhang@chem.tsinghua.edu.cn

\section{Part I}

Evaluation of sheathless interfaces (Page 2 3; Supplementary Figure 1 2)

\section{Part II}

Evaluation of non-volatile compounds deposition using Raman spectroscopy; (Page 4; Supplementary Figure 3) 


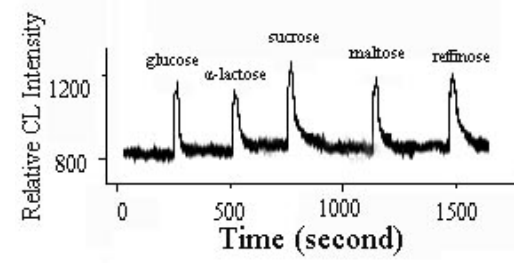

A

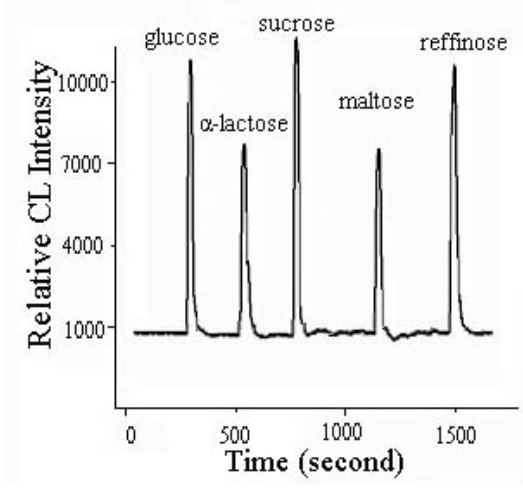

B

Figure 1. Comparison of saccharides detection using sheath liquid and sheathles technology

A: Interface with sheath liquid

B: Interface with sheathless technology

Sample information: glucose $50 \mathrm{ppm}$; sucrose, $\alpha$-lactose, maltose, reffinose, galactose and xylose $100 \mathrm{ppm}$. 

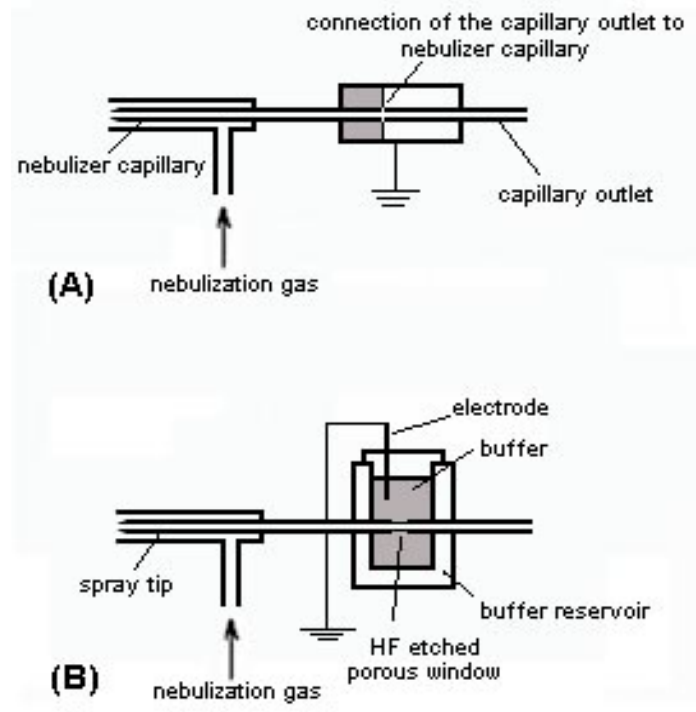

Figure 2. Schematic diagram of the home-made sheathless interfaces A: Sheathless interface based on low-dead-volume connection B: Sheathless interface based on HF etching technique 


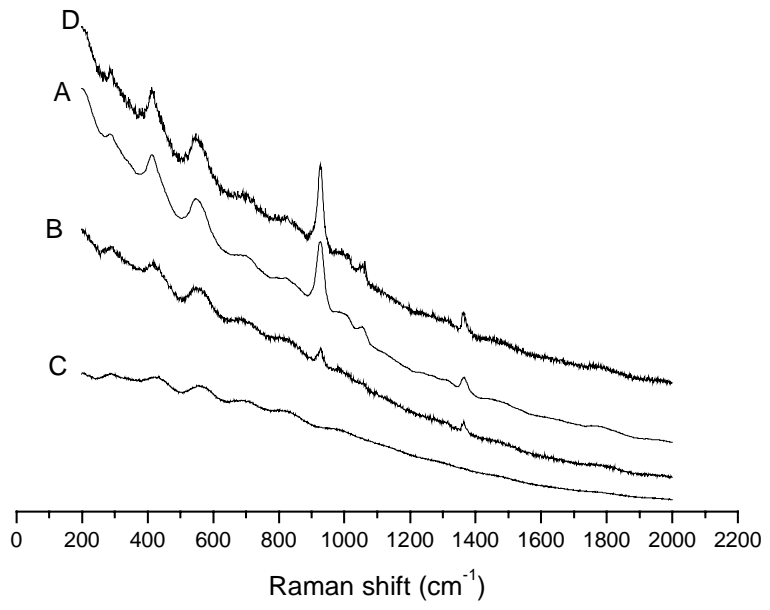

Figure 3. Raman spectra of phosphate on the surface of the porous alumina. The data obtained by a Microscopic Confocal Raman Spectrometer (Renishaw RM2000, Laser source: $785 \mathrm{~nm})$.

A: the aerosol CL detector after 100 hours test; $b$ : the aerosol CL detector after one day's test; C: the aerosol detector after washing treatment (after one day's test); D: the standard of $5 \%$ phosphate in the alumina. 